\title{
Corela
}

Cognition, représentation, langage

HS-1 1 | 2012

RJC Cotexte, contexte, situation

\section{Construction de récits et encodage de procès par des bilingues russes/français}

Tatiana Aleksandrova

\section{(2) OpenEdition}

Journals

Édition électronique

URL : http://journals.openedition.org/corela/2357

DOI : $10.4000 /$ corela. 2357

ISSN : 1638-573X

Éditeur

Cercle linguistique du Centre et de l'Ouest - CerLICO

Référence électronique

Tatiana Aleksandrova, «Construction de récits et encodage de procès par des bilingues russes/ français », Corela [En ligne], HS-11 | 2012, mis en ligne le 11 janvier 2012, consulté le 20 avril 2019. URL : http://journals.openedition.org/corela/2357 ; DOI : 10.4000/corela.2357

Ce document a été généré automatiquement le 20 avril 2019.

\section{(c) (i) (2)(2)}

Corela - cognition, représentation, langage est mis à disposition selon les termes de la licence Creative Commons Attribution - Pas d'Utilisation Commerciale - Partage dans les Mêmes Conditions 4.0 International. 


\title{
Construction de récits et encodage de procès par des bilingues russes/ français
}

\author{
Tatiana Aleksandrova
}

\section{Problématique}

1 Notre étude s'intéresse aux productions des apprenants russophones du français très avancés. Ces locuteurs adultes âgés de 25 à 35 ans ont généralement commencé à apprendre le français en Russie entre l'âge de 15 et 17 ans. Après leur arrivée en France, ils ont tous effectué des études supérieures et ont une activité professionnelle en langue seconde (L2). Le séjour minimal en France est de 5 ans. D'après la définition des niveaux de compétences en L2 de Barting (Barting, 1997), le niveau de ces locuteurs peut être considéré comme quasi-bilingue, puisque leurs productions ne sont généralement pas reconnues comme non natives par les francophones, qui signalent néanmoins l'existence de certains «traits d'étrangeté ». Pour parler de ce type de locuteurs, nous utiliserons le terme de «bilingues » dans le sens large du mot sans nous focaliser à l'état final de leur acquisition.

2 Ce groupe de bilingues présente les mêmes caractéristiques socioculturelles, toutefois il est difficile de savoir si l'exposition au français est plus intensive chez certains, que chez d'autres, si certains emploient la L2 plus régulièrement dans le contexte privé et professionnel, et d'autres, dans un de ces contextes. Nous présupposons que les locuteurs qui sont plus souvent exposés au français auront des productions en L2 moins influencées par la L1 à tous les niveaux : linguistique et informationnel, que ceux qui l'emploient dans un des contextes de la vie.

3 De plus, nous présupposons que les productions en L1 des locuteurs exposés au français de façon intensive, seront plus influencées par la L2 que celles des locuteurs utilisant la L2 dans des contextes limités. En effet, plusieurs recherches dans le domaine du bilinguisme montrent que les structures de la L2 peuvent influencer les productions en L1 des 
locuteurs bilingues vivant dans le contexte de la L2 (Cook, Pavlenko, Laufer, 2003). Ces auteurs parlent du phénomène de l'attrition, ou d'oubli de certains éléments de la L1 dans différents domaines linguistiques.

4 Afin de tester nos hypothèses sur les productions des bilingues en L2 ainsi qu'en L1, nous comparons leurs productions avec celles des russophones et francophones natifs monolingues.

\section{Cadre théorique}

\subsection{Un modèle de production langagière}

5 Un discours est produit selon les besoins communicatifs en rapport avec le type d'interlocuteur, des connaissances partagés et du contexte extralinguistique. Selon Levelt (1982), un locuteur définit d'abord ses intentions communicatives, sélectionne les informations et les linéarise, en suivant les principes de linéarisation tels que celui de l'optimisation de la connectivité, de la minimalisation des «sauts en arrière ", etc. Ces activités mentales sont appelées la phase de conceptualisation. Cette opération mène à la construction d'un message préverbal qui sera encodé linguistiquement lors de la phase de formulation. Cette phase se compose de deux étapes : l'encodage grammatical et l'encodage phonologique et conduit à la production du discours interne ou externe.

6 Le modèle de Levelt suggère donc que l'organisation de l'information au niveau conceptuel pourrait être déterminée par la structure de la langue dans laquelle s'exprime le locuteur.

7 Les résultats des études comparatives (cf. par exemple, Stutterheim von, Carroll, Klein, 2003) confirment cette hypothèse en montrant que les moyens grammaticaux diffèrent systématiquement entre les langues pour la résolution des tâches verbales complexes (description, narration ou autre). Lorsque les locuteurs de différentes langues sont soumis à la même tâche verbale, leurs productions présentent des variations dans la manière d'organiser les informations et ces variations sont en lien avec les moyens grammaticalisés spécifiques aux langues (Stutterheim von, 2003).

8 Ainsi, l'acquisition d'une L2 présuppose non seulement de découvrir les moyens grammaticaux mais aussi de comprendre à partir de ces moyens les principes organisationnels et apprendre à les mettre en œuvre dans la production d'un discours en L2. Pour le faire, les apprenants doivent abandonner les principes de gestion de l'information propres à leur L1, ce qui s'avère très difficile.

\subsection{Modèle d'analyse de discours}

Pour l'analyse des données, nous avons adopté le modèle de la Quaestio proposé par Klein et von Stutterheim (Klein, 1991). Ce modèle voit un texte comme une réponse apportée à une question globale (Quaestio) abstraite qui guide la production. Pour un type de discours comme le récit la Quaestio peut se formuler comme : 'Qu'est-ce qui se passe pour $\mathrm{P}$ (protagoniste) en T (intervalle temporel donné) ?'. Toutes les informations qui répondent directement à cette question vont fonder la trame du récit. Les autres informations comme les descriptions, les commentaires, l'expression des actions habituelles, vont représenter l'arrière-plan. La Quaestio détermine également la structure 
topique/focus des énoncés de la trame. Les éléments donnés par la Quaestio (dans ce cas le protagoniste et le temps) représentent le Topique de l'énoncé, les informations qui sont à spécifier pour un topique donné (en l'occurrence, les procès) représentent le Focus de l'énoncé. L'information se déroule d'un énoncé à l'autre dans cinq domaines référentiels ceux du temps, de l'espace, des entités, des procès et de la modalité ce qui donne lieu au maintien ou au changement de la référence.

Lorsqu'un locuteur produit un énoncé, il doit non seulement décider du contenu mais aussi prendre une décision sur l'encrage temporel pour l'événement dont il va parler.

11 Selon Klein (1994), les procès avec leurs compléments ont des caractéristiques temporelles internes, qu'on appelle aussi l'aspect lexical des verbes. Klein distingue des procès à 0 temps, à 1 temps versus à 2 temps selon que la réalisation du procès implique le passage à une situation nouvelle (Caroll, Natale, Starren, 2008).

Etant donné que les événements du film ne sont pas réels, le locuteur peut choisir la façon de les présenter comme des événements du passé ou du présent.

Nous savons que chaque événement peut être exprimé par un ou plusieurs énoncés, et que chaque procès peut être encodé par différents types de prédicats. Selon Klein, l'utilisation d'un verbe à 1 temps pour l'encodage d'un événement amène à la conceptualisation de l'événement au niveau macro, par exemple, un enfant joue dans un jardin. Lorsque, des verbes à deux états sont employés, ils présentent l'événement comme plusieurs micro-événements : un enfant prend un ballon et le lance vers une cible (Caroll, Natale, Starren, 2008). Nous empruntons la classification des prédicats de Klein (2006) pour l'analyse de l'aspect lexical.

\subsection{Différences typologiques entre les langues}

14 Les langues analysées - le français, langue romane, et le russe, langue slave, sont deux langues typologiquement différentes. Les différences pertinentes pour notre étude correspondent à la structuration des énoncés et au système temporo-aspectuel. Au niveau de la structuration des énoncés, l'ordre des constituants de la phrase est nettement plus libre en russe par rapport au français, qui privilégie la structure SVO.

Au niveau de l'expression des procès, le français possède un système verbal basé sur les temps grammaticaux : le Présent, les temps du Passé : Le Passé Composé, L'Imparfait, Le Passé Simple, Le Passé Récent, et les temps du Futur : Le Futur Simple, Le Futur Proche. Tandis que le russe, ne possède que trois temps grammaticaux : Le Présent, Le Passé, Le Futur et se base sur la distinction aspectuelle perfectif/imperfectif. Les verbes au présent correspondent aux formes imperfectives : ia chitau - je lis. Les formes du passé se divisent en imperfectives et perfectives : ia chitala - je lisais/ia prochitala - j'ai lu. Les verbes perfectifs sont généralement formés à partir de la racine verbale de l'imperfectif grâce aux préfixes: -pro, -za, -ou, etc. Les différents préfixes permettent de créer plusieurs formes perfectives et imperfectives à partir de la même racine verbale et varier la manière des actions et des mouvements ou la direction des mouvements. Par exemple, pour un verbe de mouvement hodit' - marcher, les variantes possibles sont : prihodit'/ pridti - venir, ouhodit'/ ouiti - s'en aller, zahodit/zaiti - entrer, perehodit/pereiti traverser, othodit/otoiti - s'éloigner, vihodit'/ viiti - sortir.

Nous présupposons que ces différences typologiques seront à la base de productions des locuteurs natifs et peuvent influencer le discours des bilingues en L1 et en L2. 


\subsection{Attrition} phénomènes proposée par Pavlenko (2003). Les phénomènes qui sont importants pour notre étude sont « l'emprunt » - ou addition des éléments de la L2 à la L1. Par exemple au niveau lexical, l'emprunt consiste à l'emploi des mots de la L2 dans le discours en L1 (transfert lexical). Un autre phénomène est « la modification du sens » d'un lexème de la L1 sous l'influence de la L2. Ainsi, l'extension sémantique du verbe pribivat' - arriver en russe sous l'influence du français en est un exemple. En russe cet item signifie 'l'arrivée d'un voyage', tandis qu'en français il a un sens plus large et peut être un synonyme de l'item venir. Le troisième phénomène est «le transfert de structuration », ou l'incorporation des éléments de la L2 dans le discours en L1 qui amène aux changements dans les énoncés en L1 (remplacements, simplifications) par exemple, l'emploi des pronoms possessifs selon les principes de la L2 dans le discours en L1. Le dernier phénomène, de « l'attrition » de la L1 est défini comme l'incapacité de produire certains éléments de la L1, par exemple l'emploi des structures syntaxiques incorrectes en L1 sous l'influence de la L2.

\section{Méthodologie}

Dans cette étude transversale les locuteurs sont soumis à la tâche du récit d'un film muet de 7 minutes environ appelé "Quest » mettant en scène un personnage de sable à la recherche d'eau. Il traverse différents mondes hostiles où il est menacé par les éléments de l'environnement : le papier, les pierres, les rochers qui entraînent ses chutes vers les mondes suivants. Après le visionnement du film, nos sujets sont amenés à le raconter en répondant à la question de l'enquêteur: «est-ce que tu peux me raconter ce qui s'est passé ? ». Nos informateurs ont le même âge (entre 25 et 35 ans), le même niveau d'études $(\mathrm{Bac}+4 / 5)$ et les mêmes caractéristiques socioculturelles. En revanche, 7 locuteurs sur 15 emploient le français dans le contexte familial et professionnel, tandis que les autres l'utilisent plus souvent dans le contexte professionnel que privé.

Les bilingues ont exécuté la même tâche verbale deux fois, à savoir, une fois en français et une fois en russe. Il faut signaler qu'entre les enregistrements de productions des bilingues en russe et en français il y avait toujours une interruption soit du temps (les enregistrements ont parfois étaient effectués les différents jours), soit de tâche (un intervalle temporel de trente minutes minimum de conversation libre), pour réduire l'effet de connaissance de la tâche. Parallèlement, l'ordre des langues pour l'exécution de la tâche a aussi été varié, c'est-à-dire, la moitié du groupe des bilingues a effectué cette tâche en français d'abord et dans un deuxième temps en russe, et l'autre moitié du groupe l'a effectuée en russe d'abord et après en français, indépendamment de la fréquence de l'utilisation du français dans la vie courante.

Les enregistrements sont passés dans un contexte informel, néanmoins les informateurs étaient au courrant de l'enregistrement.

Leurs récits en français et en russe ont été comparés à ceux des natifs des deux langues (groupes de contrôle) au même nombre de sujets et ayant les mêmes caractéristiques socioculturelles.

Corela, HS-1 1 | 2012 


\section{Résultats}

\section{1. Structure des récits}

Premièrement, les quatre types de productions, notamment, les récits des francophones natifs, des russophones monolingues et ceux des bilingues en français et en russe, diffèrent par leur longueur. Les récits des francophones natifs sont les plus longs parmi les autres. Ceux des russophones monolingues sont au contraire les plus courts. Les bilingues dans les deux langues produisent généralement des récits plus longs que ceux des russophones monolingues, mais plus courts que ceux des francophones. Les énoncés de la trame représentent en moyenne $50 \%$ dans toutes les productions (figure 1).

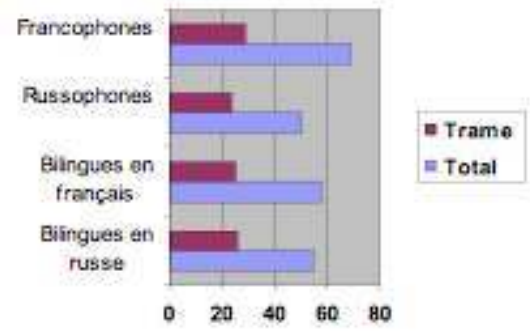

Figure 1: Longueur des productions, et longueur de la trame en nombre moyen d'énoncés

Le film met en évidence différents événements qui arrivent au protagoniste, par exemple : un bonhomme se réveille dans un désert, il se relève, il attrape une bouteille, il la secoue, il se lève, etc. Sur 33 événements répertoriés dans le film, nous avons repéré ceux qui sont encodés par au moins dix narrateurs dans chaque groupe (figure 2).

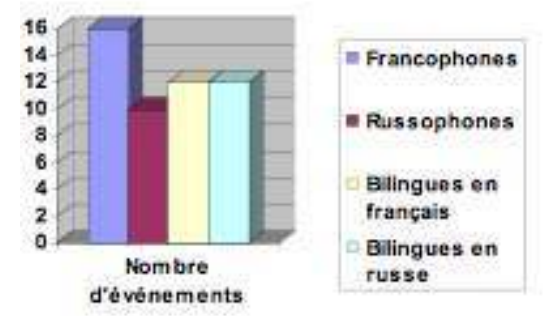

Figure 2 : Nombre d'événements encodés par au moins 10 locuteurs dans chaque groupe

La majorité des francophones encode 16 événements du film, tandis que dans le groupe des russophones ce nombre se limite par 10. Les bilingues produisant en L1 et en L2 encodent généralement 12 événements du film.

Le tableau ci-dessous (table 1) présente un exemple de productions des locuteurs monolingues. Comme on peut voir, un francophone encode 7 événements, contrairement à un russophone qui n'en encode que 4 dans la même scène du film. Dans les deux productions, les énoncés de la trame (énoncés soulignés), sont majoritaires, bien que dans la production en français il y ait deux énoncés de l'arrière-plan qui correspondent aux propositions subordonnés (table 1). 


\begin{tabular}{|l|l|}
\hline $\begin{array}{l}\text { 1. Il se réveille } \\
\text { 2. prend la bouteille } \\
\text { 3. se rend compte } \\
\text { 4. qu'elle est vide } \\
\text { 5. il se met à genoux } \\
\text { 6. et il entend de l'eau } \\
\text { 7. } \underline{\text { alors il cherche }} \\
\text { 8. d'où vient l'eau } \\
\text { 9. et il creuse le sable autour de lui }\end{array}$ & $\begin{array}{c}\text { 1. Pesochnii tchelovek pitaetsa naiti vodou } \\
\text { homme de sable essaie de trouver de l'eau }\end{array}$ \\
\hline
\end{tabular}

Le tableau suivant (table 2) montre les extraits de corpus correspondants à l'encodage de la même scène par les bilingues en français et en russe.

\begin{tabular}{|c|c|}
\hline Bilingues en français & Bilingues en russe \\
\hline $\begin{array}{l}\text { 1. Il a l'aire de se réveiller } \\
\text { 2. de se lever } \\
\text { 3. il y a une bouteille vide à coté de lui } \\
\text { 4. il la retourne } \\
\text { 5. en regardant } \\
\text { 6. s'il reste encore quelque chose } \\
\text { 7. après il regarde autour de lui } \\
\text { 8. il frotte les yeux } \\
\text { 9. et enfin il commence à faire un trou } \\
\text { dans le sable }\end{array}$ & 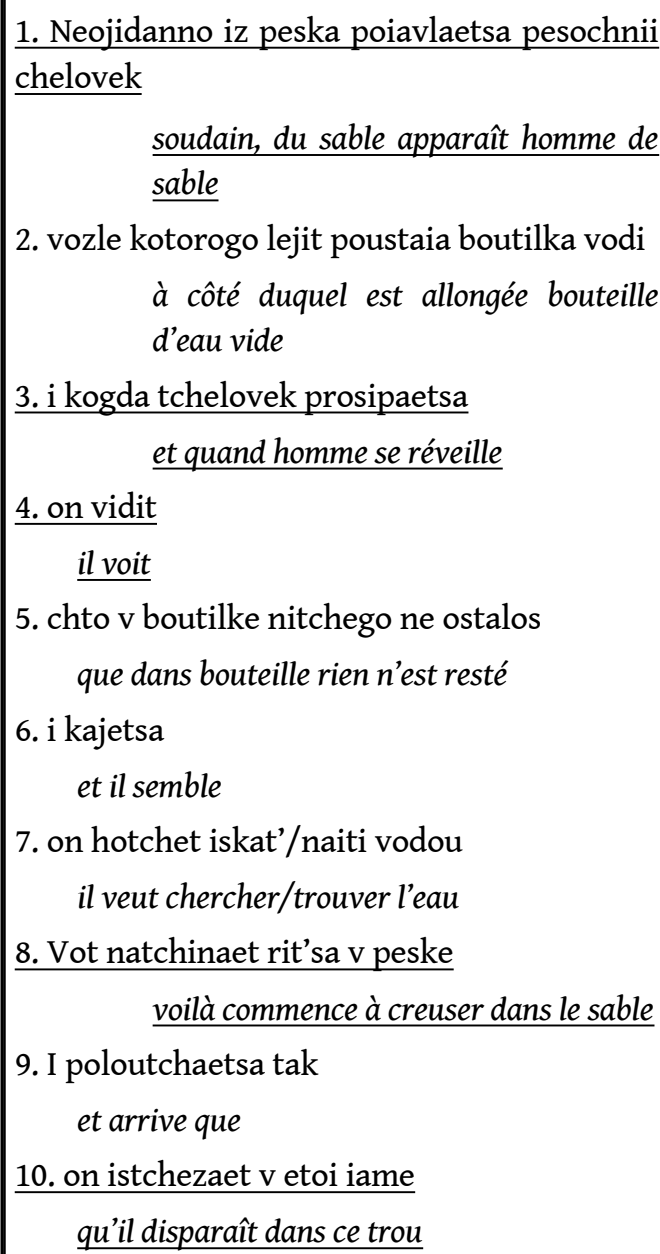 \\
\hline
\end{tabular}

Nous pouvons voir que les productions des bilingues dans les deux langues sont plus longues que celles des russophones monolingues. Ils encodent 4 et 5 événements de la même scène en français et en russe respectivement. L'arrière-plan dans leurs productions 
représente la moitié des productions en moyenne, cependant les propositions subordonnées en français sont plus rares que chez les francophones natifs.

Ainsi, les bilingues dans les deux langues produisent des récits plus longs que les russophones monolingues, en adoptant la stratégie des francophones natifs de présenter les informations comme une suite de micro-événements, ce qui n'est pas caractéristique aux russophones monolingues.

$\mathrm{Au}$ niveau linguistique, les bilingues en produisant en L1 et en L2, tout comme les russophones monolingues, emploient peu de propositions subordonnées, contrairement aux francophones natifs qui y recourent très souvent. Cette caractéristique peut être vue comme emploi de la stratégie de simplification ou le recours à la stratégie de la L1 dans la manière de présenter les informations.

Une analyse de la composition de l'arrière-plan, des choix de procès et des types de prédicats employés pour leur encodage permettra de voir si les différences quantitatives dans la longueur des productions sont liées à la façon spécifique des locuteurs de présenter les événements du film.

\subsection{Composition de l'arrière-plan}

31 Comme le montre le graphique ci-dessous, l'arrière-plan est le plus long dans les productions des francophones que dans celles des autres groupes de locuteurs (figure 3).

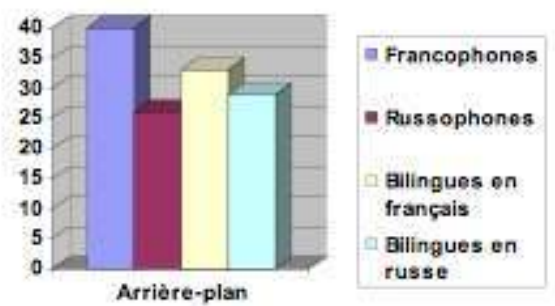

Figure 3 : Longueur moyenne de l'arrière-plan en nombre d'énoncés

L'arrière-plan se compose entre autre des commentaires, des interprétations évaluatives, des énoncés avec un prédicat extérieur et des énoncés dont le sujet grammatical est une entité autre que le protagoniste (figure 4).

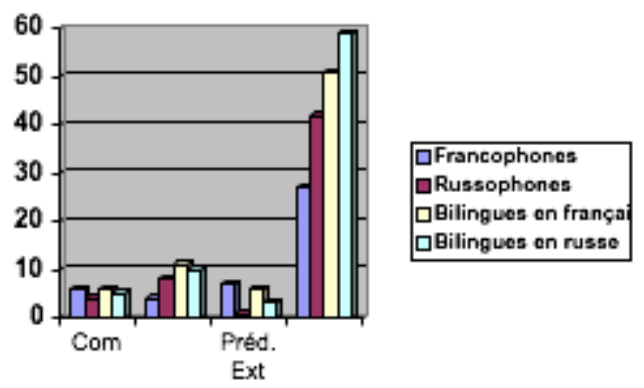

Figure 4 : Composition de l'arrière-plan

La figure 4 montre que les énoncés avec une entité du décor en tant que SN (sujet) d'une proposition indépendante ou subordonnée représentent une majorité dans les productions de tous les groupes de locuteurs. Cependant, ils sont plus nombreux dans les productions des russophones monolingues que dans celles des francophones, et 
probablement suite à cela, les bilingues en s'exprimant en deux langues, en français et en russe, leur attribuent plus souvent le statut du sujet grammatical que les francophones. commentaires. Mais le procédé auquel ils recourent le plus fréquemment, c'est l'emploi des interprétations évaluatives de l'environnement et des états physiques et psychologiques du protagoniste. Lorsqu'ils produisent en russe (L1), ils recourent aussi très souvent aux interprétations évaluatives. On peut constater que la catégorie qui occupe la deuxième place chez les russophones, reste aussi importante dans le discours des bilingues en français et en russe. En même temps, les prédicats extérieurs (verbes de perception, en particulier) sont plus nombreux dans le discours des bilingues en russe, que chez les russophones monolingues: ia vijou tcheloveka - je vois un homme, mi vidim poustinu - on voit un désert. C'est le moyen très fréquent dans les productions des francophones et des bilingues en français.

Ainsi, lorsque les bilingues produisent en français et en russe, ils emploient les mêmes stratégies que les monolingues mais dans les proportions différentes. Dans les deux langues, ils attribuent plus souvent que les francophones le statut du sujet grammatical aux entités du décor, et recourent très souvent aux interprétations évaluatives des états, ce qui est caractéristique aux productions des russophones monolingues. Ces deux caractéristiques peuvent être vues comme emploi de la stratégie de la L1 de gestion du statut grammatical des entités du décor et de la composition de l'arrière-plan du discours narratif.

Dans leurs productions en russe, les bilingues recourent plus souvent que les monolingues à l'emploi des prédicats extérieurs, qui sont caractéristiques aux productions en français.

\subsection{Référence aux procès}

Le choix du temps grammatical et des stratégies de présentation des événements sont liés avec le type des prédicats employés par les locuteurs pour l'encodage des procès. Au niveau du répertoire verbal, le nombre total des items verbaux chez les francophones est le plus élevé, d'une part à cause de l'emploi des auxiliaires être et avoir, d'autre part, puisque leurs productions sont les plus longues. Dans les productions des bilingues en deux langues, le nombre total des items verbaux est plus élevé que celui des russophones monolingues (figure 5). 


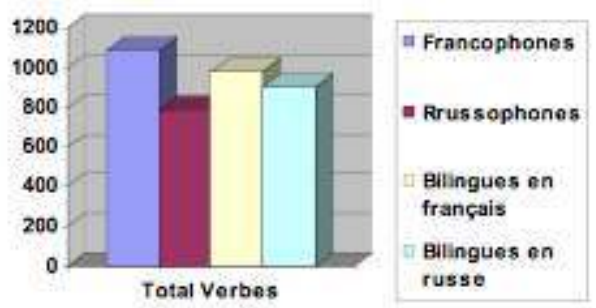

Figure 5 : Nombre moyen d'items verbaux dans les productions

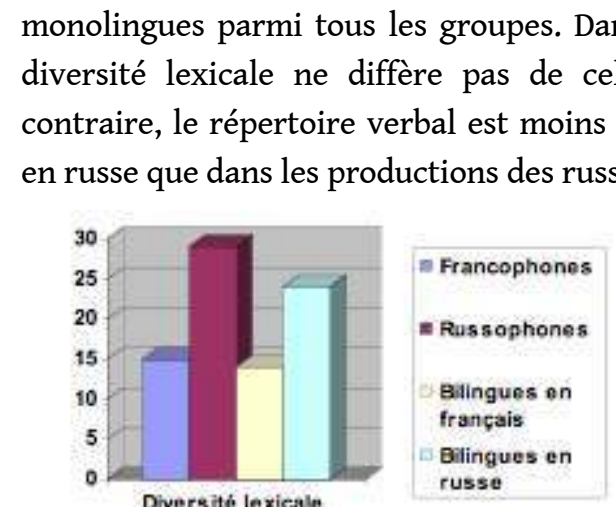

Figure 6 : Diversité du lexique verbal

Tous les items verbaux ont été divisés selon la classification de Klein (2006) en trois catégories lexicales. Les prédicats à 2 temps sont les plus fréquents dans les productions de tous types de locuteurs. Dans les productions des francophones et des bilingues en français, les prédicats à 1 temps sont presque aussi nombreux que ceux à 2 temps. Dans les productions en russe, les prédicats à 2 temps sont dominants chez les russophones monolingues, et sont un peu moins nombreux chez les bilingues. Les prédicats à 0 temps sont beaucoup plus fréquents dans les productions des deux groupes en français que dans les productions en russe (figure 7).

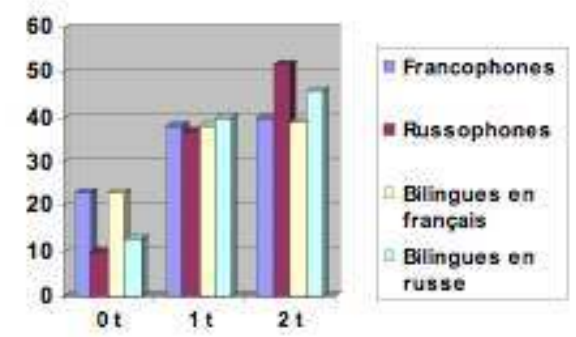

Figure 7 : Catégories lexicales des verbes

\subsubsection{Prédicats à 0 temps}

41 Le répertoire des prédicats à 0 temps ne différencie pas significativement entre les productions des locuteurs en français. Les auxiliaires être et avoir sont les verbes les plus nombreux dans cette catégorie.

42 Une des différences représente le contexte d'utilisation du verbe être par les bilingues, qui recourent souvent à l'encodage des états psychologiques du protagoniste: il est très 
étonné, il est solitaire, il est très heureux, ce que les francophones natifs font très rarement. Au contraire, comme nous avons montré dans l'analyse de l'arrière-plan, les russophones monolingues ont tendance à évaluer fréquemment les états psychologiques du protagoniste.

Ainsi, les bilingues emploient fréquemment les auxiliaires être et avoir pour exprimer les états physiques et psychologiques du protagoniste en encodant les mêmes informations que les russophones monolingues, qui sont laissées implicites dans les productions des francophones natifs.

De plus, les bilingues emploient le verbe être pour la localisation du protagoniste ou des entités plus souvent que les francophones. Les verbes lexicaux se trouver, se retrouver sont également plus fréquents chez les bilingues que chez les francophones. Cette manière de localiser les entités peut venir de la L1 des bilingues. Les monolingues russophones et les bilingues (en russe) emploient régulièrement le verbe okazivat'sa équivalent de se retrouver ( 1,5 fois/production) et ses synonymes ochutit'sa et nahodit'sa - se trouver. Les francophones natifs pour localiser les entités emploient fréquemment les prédicats extérieurs : on voit, ou la structure existentielle il y a. Ainsi, les bilingues en produisant en français suivent la stratégie de leur L1 pour localiser le protagoniste et les entités du décor.

Le russe possède un équivalent de l'auxiliaire être en français. Ce verbe existentiel russe bit - être est généralement employé dans les énoncés au passé (ia bil u vracha - j'étais chez le médecin) ou au futur (ia budu doma - je serai à la maison) et il est omis dans les énoncés au présent (ia doma - je à la maison, ia u vratcha - je chez le médecin). Il peut être employé dans les subordonnées au présent : ia vijou chto tam kto-to est - je vous qu'il y a quelqu'un là-bas, et se traduit en français par l'existentiel il y a. Une apparition de cet item au présent est attestée chez les bilingues en russe contrairement aux productions des russophones monolingues:

$$
\begin{aligned}
& \text { a. I vdroug vidit opiat } \\
& \text { Et soudain il voit de nouveau } \\
& \text { b. Chto nepodalekou opiat est loujitsa vodi } \\
& \text { Que à côté de nouveau est (il y a) une flaque d'eau }
\end{aligned}
$$

Ce phénomène peut être vu comme un transfert de structuration de l'énoncé de la L1 sous l'influence de la L2.

47 Le répertoire des verbes à 0 états est moins riche dans les productions des bilingues en français, et les stratégies d'utilisation des ces items sont souvent prédominées par la L1 (encodage de la localisation, expression des états psychologiques).

Il est intéressant de noter que le répertoire verbal des bilingues en russe diffère de celui des russophones monolingues dans l'emploi plus fréquent du modal moch - équivalent de pouvoir assez fréquent en français et presque absent chez les russophones monolingues, et dans l'emploi d'un grand nombre de verbes de cognition (comme chez les francophones natifs), ce qui n'est pas caractéristique aux productions des russophones monolingues.

49 Un exemple de transfert de structuration montre également que la maîtrise de la L2 influence le répertoire verbal et les stratégies communicationnelles des bilingues dans les productions en L1. 


\subsubsection{Prédicats à 1 temps}

50 La diversité lexicale pour cette catégorie est plus importante dans les productions en russe qu'en français. Pour l'illustrer nous observons le répertoire verbal employé pour l'encodage d'une des scènes exprimées par la majorité des locuteurs où le

\begin{tabular}{|c|c|c|c|}
\hline Francophones & $\begin{array}{l}\text { Bilingues en } \\
\text { français }\end{array}$ & Russophones & Bilingues en russe \\
\hline Creuser (10) & Creuser (9) & kopat' (9)- creuser & kopat' (13) \\
\hline Gratter (3) & Gratter (1) & $\begin{array}{l}\text { raskapivat'/raskopat' (1) - } \\
\text { fouiller }\end{array}$ & raskapivat (1) \\
\hline \multirow[t]{6}{*}{ Fouiller (2) } & & $\begin{array}{l}\text { dokapat'sa (1)- creuser } \\
\text { jusqu'au fond }\end{array}$ & \\
\hline & Faire un trou (2) & rit' (4)- bêcher & rit' $^{\prime}(\mathrm{sa}) 14$ \\
\hline & & $\begin{array}{l}\text { gresti' (1)- creuser avec les } \\
\text { mains }\end{array}$ & gresti \\
\hline & & $\begin{array}{l}\text { otgrebat' (1) - creuser pour } \\
\text { éloigner }\end{array}$ & \\
\hline & & & $\begin{array}{l}\text { razgrebat' } \\
\text { déblayer }\end{array}$ \\
\hline & & & $\begin{array}{l}\text { raschirat' (1) - } \\
\text { élargir }\end{array}$ \\
\hline 3 & 3 & 6 & 6 \\
\hline
\end{tabular}

51 protagoniste creuse un trou dans le sable (table 3).

Table 3 : Répertoire verbal pour le procès « creuser le sable »

52 Le répertoire verbal est plus riche en russe qu'en français grâce à la variabilité des préfixes qui créent de nouveaux verbes à partir de la même racine verbale, comme : kopat' - creuser, raskapivat' (imperfectif)/ raskopat' (perfectif) - creuser, dokapivat'sa (imperfectif) - creuser jusqu'au fond; ou gresti (imperfectif) - creuser avec les mains, otgrebsti (perfectif) - creuser avec les mains pour éloigner quelque chose. Néanmoins, les verbes les plus fréquents en russe sont kopat'- qui peut signifier 'creuser avec les mains', mais plus précisément l'action de 'creuser avec une pelle', et rit' - équivalent direct de 'creuser' en français. Les russophones monolingues emploient plus fréquemment le premier item kopat, tandis que les bilingues recourent majoritairement à l'équivalent de creuser en français - rit'. Cette tendance peut être vue comme une stratégie des bilingues d'employer les moyens linguistiques, ici les moyens lexicaux, similaires ou partagés par deux langues. 

bilingues en russe pour le choix du complément des verbes sous forme de pronoms personnels toniques :

1. I zatchem to natchinaet raskapivat pesok priamo pered nim Et commence à creuser le sable devant lui

2. Riadom s nim on ouvidel boutilkou

A coté de lui il a vu une bouteille

Dans ces exemples, après un verbe à la 3ème personne du singulier les locuteurs emploient un pronom personnel complément à la 3ème personne du singulier également, en employant la stratégie du français, tandis que le choix approprié en russe serait le pronom à la 1ère personne du singulier soboi - soi.

\subsubsection{Prédicats à 2 temps}

La majorité des procès est encodée par les verbes à 2 temps dans toutes les productions. Un des procès le plus fréquent, rencontré dans les trois épisodes du film et encodé par tous les locuteurs, est «le bonhomme tombe dans un trou du sable, du papier ou de pierres ». Les francophones natifs ont tendance à encoder ce procès par deux énoncés. Cependant, certains locuteurs l'encodent par un seul énoncé. Dans ces cas, ils recourent à l'emploi du passif : il est englouti en encodant ainsi le résultat de l'événement.

Les russophones monolingues, au contraire, recourent à cet événement plus souvent par un seul énoncé et utilisent pour cela une phrase active : on provalivaetsa. Le verbe de mouvement provalivat'sa n'a pas d'équivalents en français, il encode le mouvement de 'tomber à l'intérieur de quelque chose'. L'item tomber en français exprime le mouvement vers le bas, sans préciser si ce mouvement se passe sur la surface, du haut vers le bas, ou à l'intérieur de quelque chose.

Les bilingues, en produisant en deux langues, encodent plus souvent cet événement par deux énoncés en divisant la scène en micro-événements, suivant ainsi la stratégie des francophones natifs.

Cependant, lorsqu'ils encodent cet événement par un seul énoncé, ils emploient les phrases actives, contrairement aux francophones, en employant le verbe tomber en français et provalivat'sa - 'tomber à l'intérieur de quelque chose', en russe et suivent ainsi la stratégie de leur L1.

Dans les cas, où les bilingues encodent cette scène par deux énoncés, ils procèdent de la même manière que les francophones natifs, le premier énoncé met en scène une entité inanimée : le sable l'emporte, et le deuxième - exprime le résultat : et il tombe.

Comme nous l'avons indiqué, l'item russe provalivat'sa est plus spécifique que le verbe tomber en français, puisqu'il indique le mouvement vers le bas à l'intérieur d'un espace. Ainsi, les russophones, soit n'emploient pas de compléments pour ce verbe, soit le complément est attaché par la préposition $\mathrm{v}$ - dans : on provalivaetsa v sleduushii mir - il tombe dans le monde suivant.

61 Les francophones accompagnent souvent l'item tomber d'un complément attaché par la préposition à travers : il tombe à travers le sable.

Dans les productions des bilingues en russe, le verbe spécifique provalivat'sa est parfois accompagné de compléments attachés par la préposition tcherez, équivalent de à travers 
en français : vdroug provalivaetsa cherez pesok - il 'tombe à l'intérieur' à travers le sable. L'emploi de cette préposition est un cas de transfert de structuration du français sur le russe.

Le russe possède un item équivalent de tomber en français - padat, il est souvent employé dans les productions. Les russophones monolingues l'emploient surtout pour encoder l'atterrissage du protagoniste dans un nouveau monde. Les francophones natifs encodent ce procès plus rarement, lorsqu'ils le font, ils emploient généralement l'item arriver. Les bilingues en produisant en deux langues encodent régulièrement ce procès en suivant la stratégie de leur L1. En produisant en français, ils emploient l'item tomber pour encoder l'atterrissage du personnage. Dans quelques productions des bilingues en russe, nous retrouvons l'emploi de l'équivalent du verbe français 'arriver' - pribivat': v etom epizode ia tak ponimau on pribivaet iz predidoushei stseni - dans cet épisode d'après ce que je comprends il arrive de la scène précédente, ce qui n'est jamais attesté chez les russophones monolingues. Effectivement, le verbe pribivat' - arriver en russe a le sens d'arriver d'un voyage. Cet emploi du verbe pribivat' en russe peut être considéré comme un cas d'extension sémantique.

Ainsi, les russophones ont tendance à encoder les événements par un seul énoncé, contrairement aux francophones qui préfèrent les diviser en sous événements encodés par plusieurs énoncés. Au niveau du choix des catégories lexicales des verbes, les russophones et les francophones recourent aux verbes à 1 et à 2 temps indépendamment du fait qu'ils utilisent un ou plusieurs énoncés pour l'encodage des actions. Un verbe de 2 temps comme tomber peut être utilisé séparément ou accompagné d'un autre item pour l'encodage de l'événement "le bonhomme tombe dans un trou». Au niveau des stratégies, les bilingues en produisant en L2 dans certains cas, suivent la stratégie de leur L1 en encodant les événements par un seul énoncé, dans d'autres cas, ils suivent la stratégie de la L2 en divisant les événements en sous événements. Lorsqu'ils produisent en russe, contrairement aux russophones monolingues, ils ont tendance à présenter les événements comme une suite de micro-événements en employant les verbes à 1 et à 2 temps.

65 Au niveau lexical, le répertoire verbal est plus riche dans les productions en russe qu'en français. Le répertoire verbal des bilingues produisant en russe est assez varié. Néanmoins, le choix des verbes et leurs compléments reflète souvent la connaissance et l'emploi régulier du français.

\section{Discussion et conclusion}

Les comparaisons interlingues montrent que les locuteurs monolingues : francophones et russophones ont des manières différentes pour présenter des informations. Les francophones natifs ont tendance à encoder les événements dans l'ordre dans lequel ils se sont produits en employant plusieurs énoncés de la trame ou de l'arrière-plan pour présenter un événement, ce qui conduit à un découpage des événements en sous événements. Au contraire, les russophones monolingues encodent moins d'événements du film et les expriment plus souvent par un seul énoncé, qui fait habituellement partie de la trame, ce qui conduit à la présentation des événements au niveau macro. Cette différence se retrouve dans les productions des bilingues. 
67 Les études précédentes ont montré que les apprenants même de niveaux très avancés conceptualisent le discours selon les principes organisationnels de leur L1 (Lambert, Carroll, Stutterheim, 2008). Cependant, les bilingues russes/français en produisant dans les deux langues découpent certains événements en micro-événements, et en encodent d'autres au niveau macro. Dans tous les cas, leurs productions dans les deux langues sont plus longues que celles des russophones monolingues et reflètent en partie les principes de découpage de l'information propres à la L2.

$\mathrm{Au}$ niveau de la conceptualisation du message en L1 et en L2, la quantité de l'exposition des bilingues au français ne semble pas avoir d'importance significative. Ainsi, l'hypothèse que les locuteurs utilisant le français plus intensivement dans leur vie, construiraient le discours en L2 différemment que ceux qui l'utilisent dans des contextes plus limités, ne se justifie pas. Il en est de même pour la phase de la formulation en L2. Les bilingues partagent les mêmes caractéristiques. Leur discours est souvent influencé par les principes de la formulation des énoncés de la L1, cela est visible dans l'emploi de l'auxiliaire être pour l'expression des états psychologiques et des localisations, la formulation des phrases actives et le nombre restreint des propositions subordonnées.

Ces résultats confirment les résultats des études précédentes qui indiquent que les apprenants même aux stades très avancés emploient les moyens linguistiques de la L2 selon les principes informationnels de la L1 (Lambert, Carroll, Stutterheim, 2008).

En revanche, les productions en L1 des bilingues qui sont plus intensivement exposés au français sont plus influencées par la L2 au niveau grammatical. Ces influences sont visibles :

71 a) dans les cas de modification du sens des items, notamment dans l'extension sémantique du verbe pribivat' - 'arriver' en russe, qui, sous l'influence du français, est employé par les bilingues comme synonyme du verbe venir. Cette utilisation n'est pas appropriée en russe ;

72 b) au niveau de la construction des énoncés, les cas de transfert de structuration, en particulier, l'emploi des prépositions russes selon les principes du français ;

73 c) finalement, l'emploi des pronoms toniques en L1 selon les règles de grammaire de la L2, selon la classification de Pavlenko (2003) représente un phénomène d'attrition de la L1.

74 Ainsi, le cotexte de productions des bilingues en russe, qui sont plus souvent exposés au français, reflète la maîtrise et l'emploi fréquent d'une L2. Le cotexte de la L1 de productions des locuteurs bilingues qui utilisent le français moins souvent dans leur communication quotidienne est moins influencé par les structures de la L2. Ainsi, l'utilisation très intensive d'une L2 conduit aux effets plus nombreux de cette langue sur la L1 de locuteur.

75 Ces résultats vont dans le même sens avec les analyses de Pavlenko (2003) des bilingues russes/anglais dans le sens où elle trouve les phénomènes d'attrition de la L1 dans les mêmes domaines linguistiques du russe.

76 En conclusion, on peut constater que le discours de bilingues en deux langues n'est pas identique à celui des monolingues (russes et français) et présente son propre système de fonctionnement qui reflète la connaissance des deux langues, ce que Cook (2003) appelle la 'multi compétence'. 

bilingues pour l'expression d'autres tâches verbales, dans différentes situations de communication.

\section{BIBLIOGRAPHIE}

BARTING, I. (1997) : « L'apprenant dit avancé et son acquisition d'une LE », Aile n 9, pp. 10-50. CAROLL, M. NATALE, S. STARREN, M. (2008): « Acquisition du marquage du progressif par des apprenants germanophones de l'italien et néerlandophones du français ", Aile n 26, pp. 11-31. соOK, V. (2003): Effects of the Second Language on the First, Multilingual Matters, Clevedon. KLEIN W. STUTTERHEIM C. von (1991): « Text structure and referential movement », Sprache und Pragmatik, Vol. 22, pp. 1-32.

KLEIN, W. (1994): Time in Language, Routledge, London.

KLEIN, W. (2006): “On finiteness” (éd.) van Geenhoven, V. Semantics in Acquisition, Springer, Dordrecht, pp. 245-272.

LAMBERT M. (2006) : « Pourquoi les apprenants adultes avancés ne parviennent-ils pas à atteindre la compétence des locuteurs natifs »? (éd.) Engwall, G. Construction, acquisition et communication : études linguistiques de discours contemporains, Université de Stockholm, pp. 151-170.

LAMBERT, M. CARRoll, M. STUTTERHeim, Ch. von (2008) : « Acquisition en L2 des principes d'organisation de récits spécifiques aux langues ", Aile nº 26, pp. 12-28.

LAUFER, B. (2003) : « The Influence of L2 on L1 Collocational Knowledge and on L1 Lexical Diversity in Free Written Expression ", (éd.) cooK, V. Effects of the Second Language on the First, Multilingual Matters, Clevedon, pp. 19-32.

LEVELT W.J.M. (1982): « Linearisation in describing spatial networks », (éd.) HATTORI S. INOUE K. Proceedings of the XIIIth International Congress of Linguistics, Tokyo-La Haye:CiPh.

PAVLENKo, A. (2003): 'I feel Clumsy Speaking Russian': L2 Influence on L1 in Narratives of Russian L2 Users of English, (éd.) cooK, V. Effects of the Second Language on the First, Multilingual Matters, Clevedon, pp. 32-62.

STUTTERHEIM, C. von (2003): « Linguistic structure and information organisation: the case of very advanced learners ", (éd.) FOSTER-COHEN, S. PEKAREK DOEHLER, S. EUROSLA yearbook, John Benjamins P.C. The Netherlands.

STUTTERHEIM, C. von, CARROLL, M. KLEIN, W. (2003): « Two ways of construing complex temporal structures ", (éd.) LENZ, F. Deictic Conceptualisation of Space, Time and Person, John Benjamins Publishing Compagny, pp. 98-133. 


\section{RÉSUMÉS}

Notre étude s'intéresse aux productions en français (L2) et en russe (L1) de quasi-bilingues russes/français. D'un côté, leur discours en deuxième langue nous paraît intéressant en raison des divergences par rapport aux productions des francophones natifs dans la manière de présenter les informations. En effet, plusieurs études comparatives dans le domaine de l'acquisition des langues montrent que les apprenants, même de niveaux très avancés, organisent le discours en L2 de façon différente que les natifs (Lambert, 2006). De l'autre côté, les productions des quasi-bilingues en première langue ne sont pas identiques à celles de russophones monolingues. Nous essaierons de démontrer ces influences linguistiques à travers les productions orales de type narratif dans les deux langues.

This study is devoted to an analysis of near-native speakers' productions. We presuppose that not only the First Language (L1) influences the productions in the Second Language (L2), but the L2 can also affect the productions in the L1. The oral discourse in French by Russian/French nearnative speakers is influenced by the L1 in the area of back-ground composition, and linguistic choices. The productions in the L1, differ from that of Russian monolinguals in the area of event representation and in the organisation of elements in the utterance. The cases of linguistic trasfer are visible in the both languages.

\section{INDEX}

Keywords : Transfer, translinguistic influences, information structure, lexical aspect, event representation.

Mots-clés : Transfert, influences translinguistiques, structure informationnelle, aspect lexical, représentation des événements.

\section{AUTEUR}

\section{TATIANA ALEKSANDROVA}

Université Paris 8 - Vincennes, Saint-Denis 